\title{
Facilitating decision-making by choosing an NDT method based on information need
}

\author{
Anna Ericson Öberg ${ }^{1,2} \cdot$ Peter Hammersberg ${ }^{1}$
}

Received: 22 October 2015 / Accepted: 9 May 2016 / Published online: 19 May 2016

(C) The Author(s) 2016. This article is published with open access at Springerlink.com

\begin{abstract}
This paper discusses the choice of an NDT method (non-destructive testing) from an information need perspective. It bridges the gap between technical methods, monitoring procedures and the need for information in an industrial application. The simple pull approach described starts from the decision that needs to be taken rather than from a previous data push procedure. That makes it more likely to match the method with the actual need, which leads to more effective and efficient evaluations. The pull approach is described by using a case from the welding industry, evaluation of the weld toe radius. The toe radius, defining how smooth the transition between the plate and the weld is, has been found critical to fatigue strength. Depending on the internal customer's need of information, various evaluation solutions are preferred. The methods range from manual gauges to fully automated camera solutions. An interview study performed shows that the current process for choosing evaluation method differs. The person needing the information is often not the starting point in the process. A change in starting point influences both the choice of NDT method and visualization and thereby how efficient and effective the decision-making support will be. The financial effect is significant.
\end{abstract}

Keywords (IIW Thesaurus) Weld toes · Quality assurance · Non destructive testing $\cdot$ Information

Recommended for publication by Commission V - NDT and Quality Assurance of Welded Products

\section{Anna Ericson Öberg}

anna.ericson.oberg@volvo.com

1 Chalmers University of Technology, Gothenburg, Sweden

2 Volvo Construction Equipment, Box 303, SE 67127 Arvika, Sweden

\section{Introduction}

Prasad and Nair [1] state that non-destructive test and evaluation is aimed at extracting information on the physical, chemical, mechanical or metallurgical state of materials or structures. Research on non-destructive testing (NDT) and welding is, however, primarily focused on the weld process itself or the use of a specific technology for assessing the quality and not on the surrounding data handling structures. Searching for "choice of NDT" returns only four articles in Scopus, none in Web of Science and seven on ndt.net. Several authors have described the suitability of different NDT techniques for detecting certain defects. Raj et al. [2] state that before selecting an NDT method, the user must know what type of defects to expect. POD (probability of detection) is also commonly mentioned. The knowledge of the POD curves of specific flaws in specific testing conditions using defined inspection methods is of course important, however in a later stage of the process. Commonly, it seems to be assumed that the purpose of the evaluation is already known. It could be that different functions of the company focus on their own area, no one taking the lead in the overall purpose discussion.

This paper highlights and bridges the gap between the technical methods and the need for information occurring in the industrial application. The likelihood of achieving a more effective and efficient combination of methods, tailored data visualization and process monitoring increases when using a pull approach. This will be exemplified by an application to a real case. Results from an interview study will also be presented.

The reason for testing is not the test itself but to get information that can be used as decision-making support. As easy as it may sound, that is not obvious when transferring information and knowledge across a multi-functional operation. Especially for a specialist in a certain method, the focus easily 
Fig. 1 The process of the pull approach with an information need perspective $[3,4]$

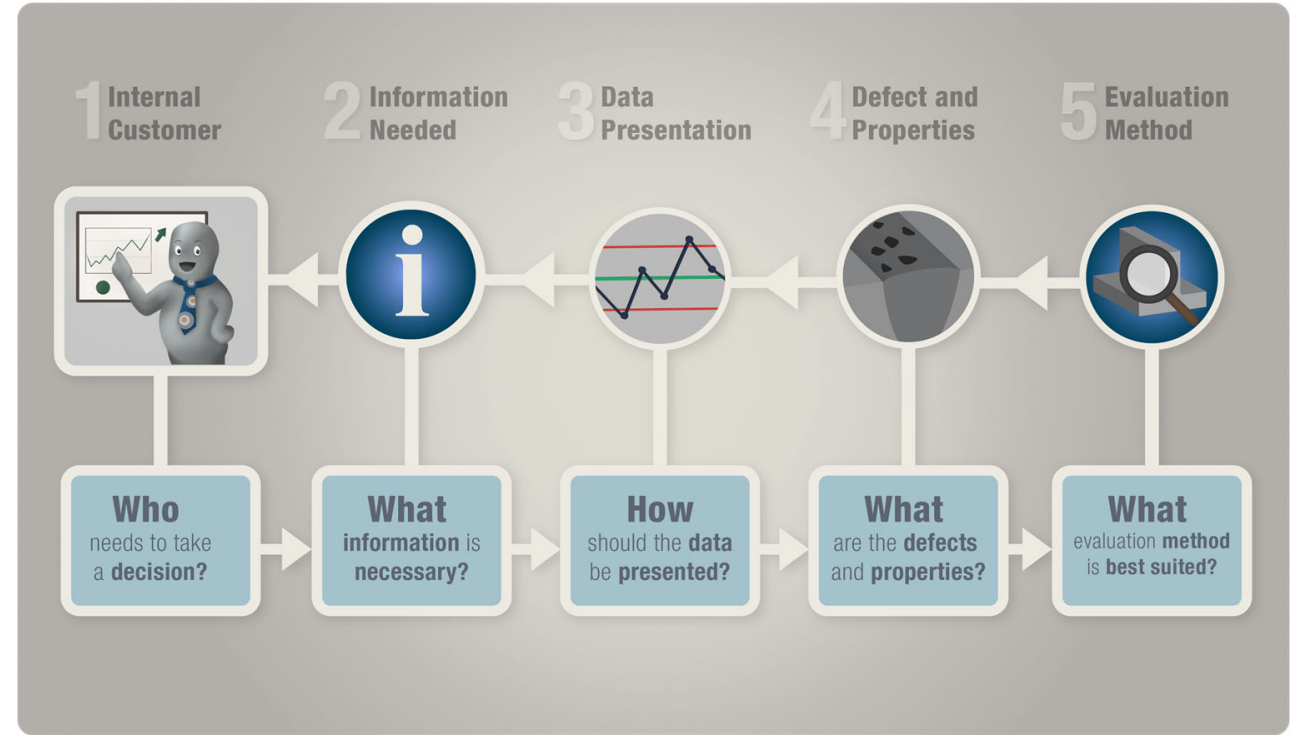

ends up in the details of the equipment available, limitations, etc. However, there are many benefits such as improved information precision and reduced set-up and operation times that will reduce total cost if the order of thinking is reversed, as suggested in the pull approach in Fig. 1. The first step is to start reflecting on who is going to make a decision.

The second step in the pull approach is to define what information is needed to make this decision. The third step is to consider the question how the data are best analysed and presented to support the decision-making. It is not until the fourth step that it is time to question what defects or qualities we are looking for. Finally, when all these issues have been resolved, we need to define what method is best suited to obtain the information. By thinking information-driven evaluation, the choice of the evaluation method depends on what is best suited for the needs of the "internal customer" and not, e.g., the most technologically advanced equipment.

\section{Empirical study}

In manufacturing, there are many different professions and roles that have different information needs to make decisions. The welder wants to know if the part he or she just welded is OK to send to the next station. The programmer is interested in whether the robot program will deliver products within the specification limits or not. The designer is concerned about what requirements he or she can impose on the drawing depending on the manufacturing methods used. The manager, on the other hand, needs information about how improvement resources can best be allocated. Jonsson et al. [5] argue that production technology researchers and structural design researchers have had only limited dialogue and each group has focused on its own narrow field of interest. This has, according to Jonsson et al., led to inconsistencies in the definition of so-called "weld class systems", which results in little or no relation to the actual performance of the welded structure.

In the following example, a requirement that all the different people need to relate to is the weld toe radius, also called transition radius. The weld toe radius defines how sharp the transition between the weld and the base metal is (see Fig. 2).

Kassner et al. [6] state that weld quality, e.g., the real weld toe radii, has a significant influence on fatigue strength. According to Björk et al. [7], design criteria and requirements depend on the primary function of the joint. In terms of fatigue strength, the weld flank angle and smoothness of the weld toe transition will greatly influence the quality of especially a load-carrying joint. Therefore, a new recommendation regarding weld quality must make a distinction between joint functions.

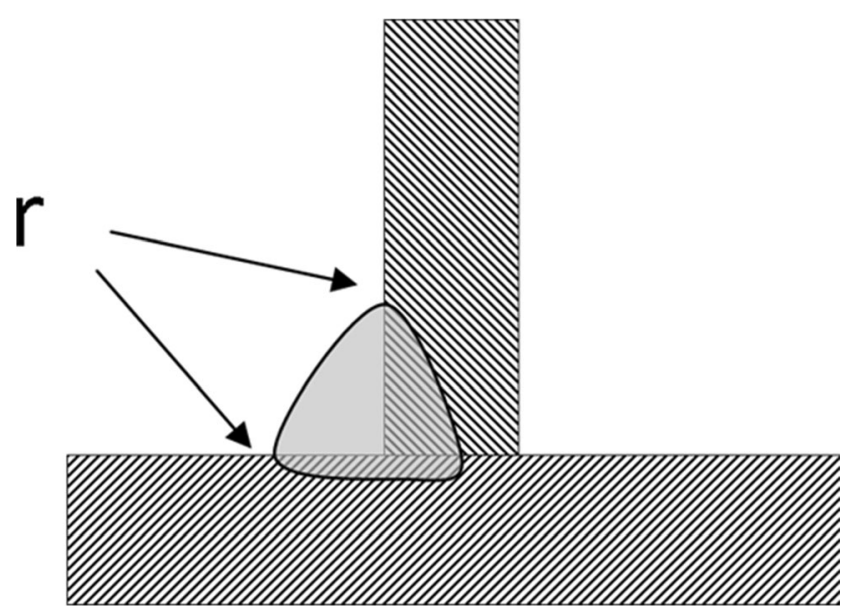

Fig. 2 Illustration of weld toe radius 
Fig. 3 Radius gauges for assessing the weld toe radius

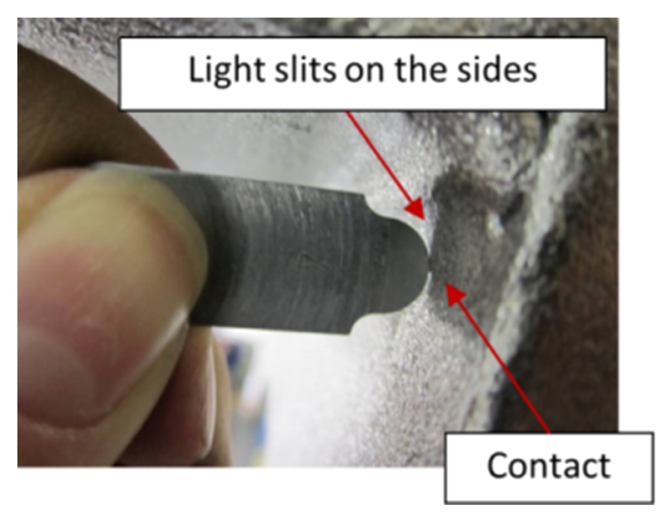

Since the radius has shown to have strong influence on fatigue life according to, among others, Lee et al. [8] and Jonsson et al. [5], it is therefore included in corporate weld standards, such as Volvo standard 181-0004 [9]. Since this demand is rather company-specific and not included in the common standard ISO 5817 [10], there is also no existing common evaluation method used. Wide combinations of the radius and angles can occur (Pang [11]) and be evaluated in several different ways (Harati et al. [12]).

To put this into context, an example from a heavy-welding industry of construction equipment will be used. The products manufactured are built on large, welded structures exposed to fatigue, where the toe radius is influential. The pull approach, in contrast to the former data push procedure, will be illustrated by three scenarios. To get a broader view, results from an interview study including 19 plants conducting welding will be presented.

\subsection{Scenario 1-the welder}

The welder needs to know if the weld is approved to pass on or not. The welder does not need to know the size of the radius, only if it is within the limits of the weld class or not. It should be a simple go/no-go decision; precise values are not necessary. The easiest way to fulfil this information requirement is simple radius gauges, described in Fig. 3. The gauge is placed against the weld toe radius, and depending on the light slit appearance, it can be ascertained whether the weld toe radius is larger or smaller than the radius of the gauge.

\subsection{Scenario 2-the programmer}

The programmer has a different information need. The programmer needs to know how the program is working, if it will produce welds within the specification limits and the spread of the result. The focus is on the process rather than on individual products.

This may mean that it is necessary to think a little extra about how the results should be presented. Control charts are often a good way to provide information about the process. Figure 4 illustrates the common components that are present in a control chart. A control chart consists of a centre line indicating the average as well as upper and lower control limits. The limits are statistically calculated based on past

Fig. 4 Example of control chart

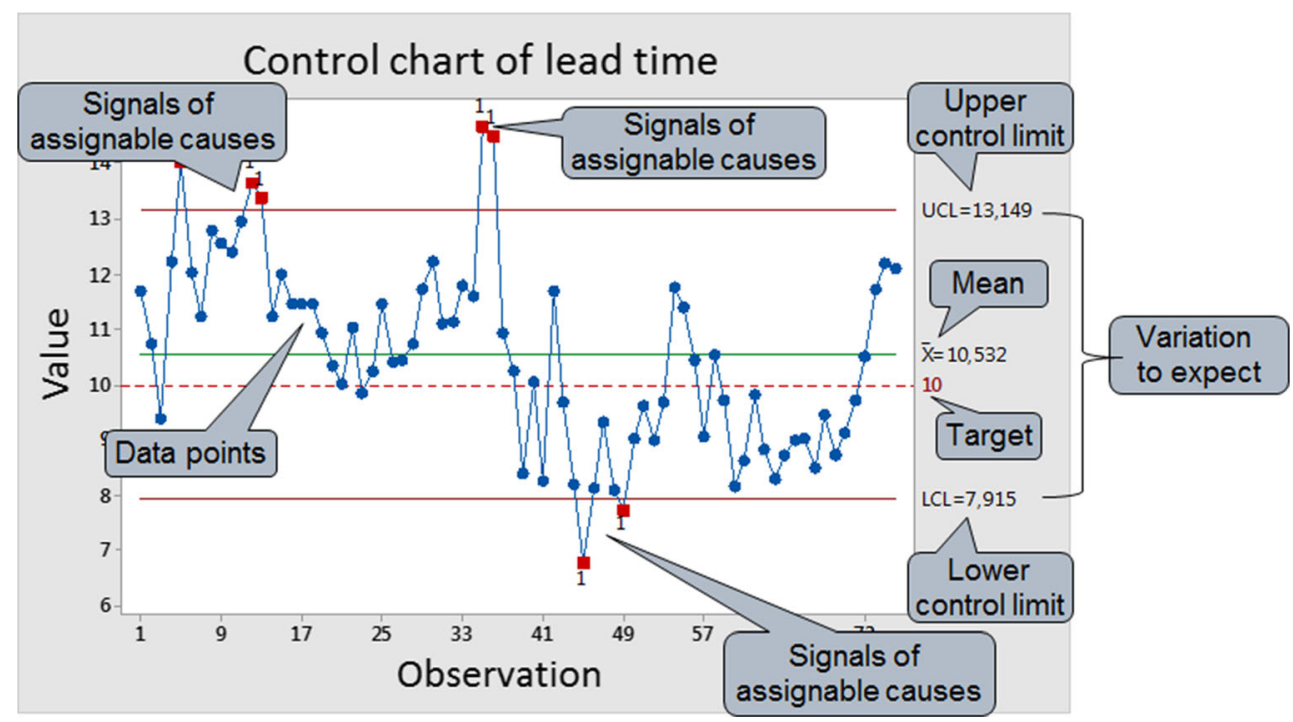


Fig. 5 Control chart displaying weld toe radius

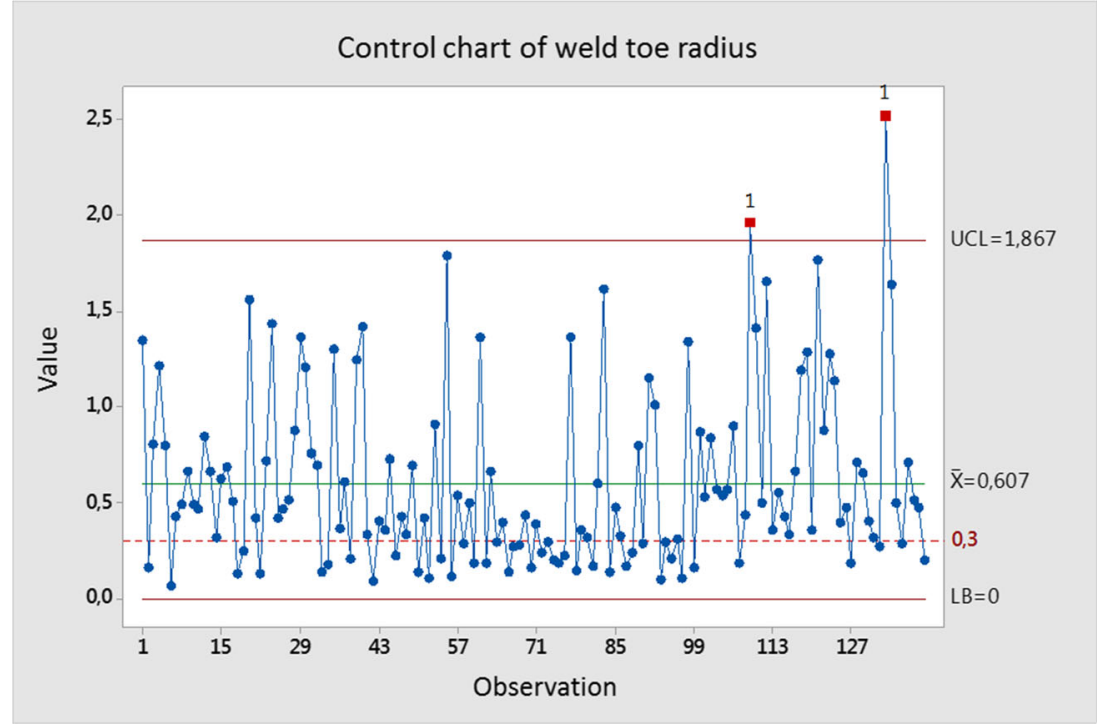

performance. When a sample falls outside the control limits, it indicates an assignable cause, meaning the process is unstable. A control chart indicates the right type of action, depending on whether the process is found to be stable or unstable, capable or not, etc. The strength of using control charts to further the discussion and common understanding within the operation from output go/no-go to process variability has a long history and has been studied by, among others, Danielsson and Holgård [13], Deming [14] and Wheeler [15].

Figure 5 shows a control chart displaying the weld toe radius for a certain weld. The weld toe radius varies considerably and the welding is not a stable process. Some radii are outside the specification limits as well, which means rework. Worth noticing is that on average the radii are $\mathrm{OK}$; the problem is the variation.

If the programmer needs to obtain the value of the radius in order to compare the results of different programs, WIA (weld impression analysis) is an option. The method is described by Ericson Öberg [3] and Harati et al. [12]. Figure 6 shows the process. A two-component polymer is applied to the weld surface and left to solidify for a few minutes. The impression is removed from the weld surface, cut and placed in a microscope. Help lines representing the plate are drawn and finally a circle is fit to the radius between the lines.

If the information need of the programmer is the variation along the weld, laser scanning could be a possibility. By projecting a laser line on the weld, a scanned profile is obtained including the weld toe radius described by, for example, Lindgren and Stenberg [16]. This equipment, shown to the left in Fig. 7, is not currently used at the company but is an example from the research project Onweld, funded by Vinnova.

To be able to control the process, information has instead to be acquired earlier, already during the process. In this case, a CCD and IR camera was used to collect information about the performance during welding, shown to the right in Fig. 7. This information could then be used to adjust the process. This equipment is also only at the research stage as part of the finished Faromonita project, funded by the Knowledge Foundation.
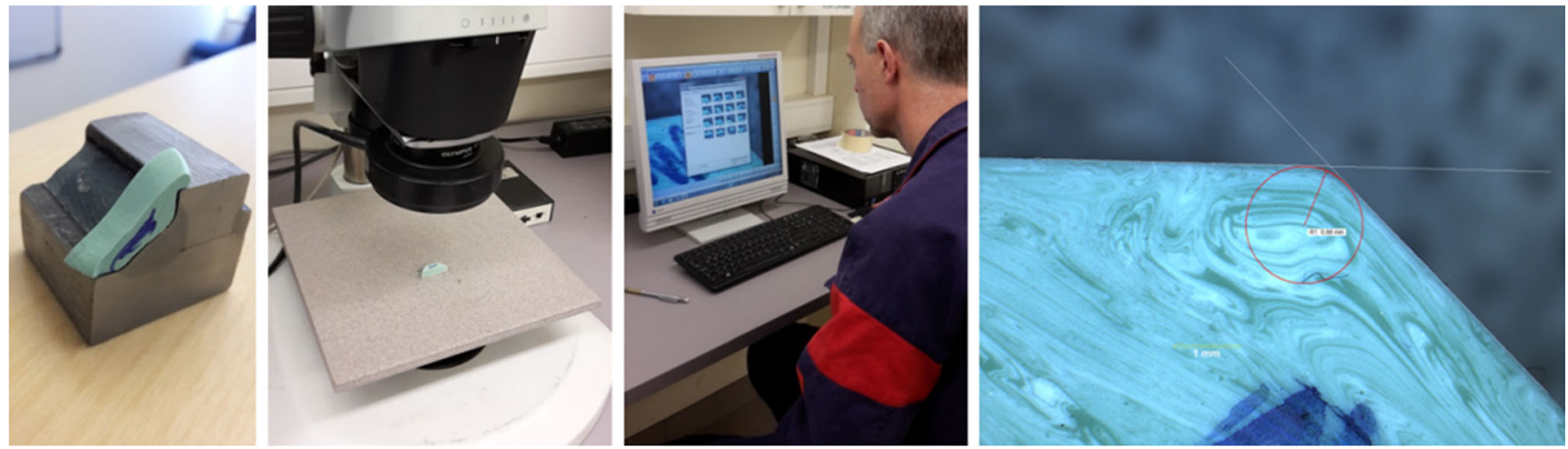

Fig. 6 WIA (weld impression analysis) used for evaluating the weld toe radius 
Fig. 7 Laser scanning of the weld geometry during (right picture) as well as after welding (left picture)
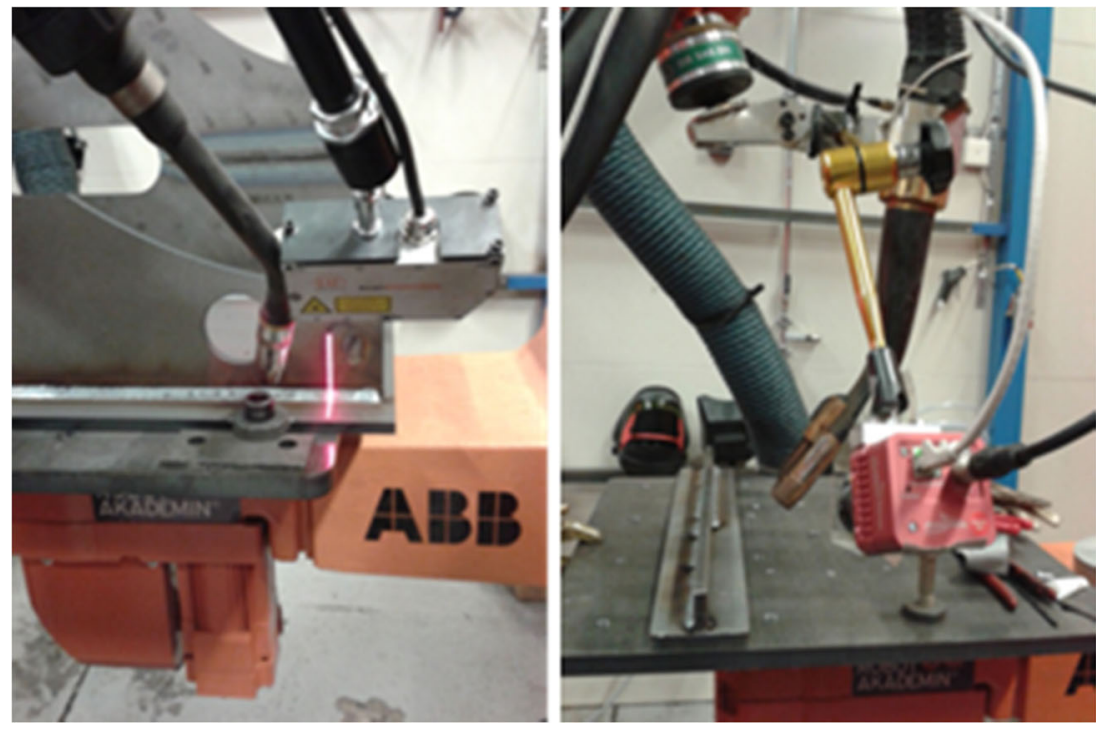

\subsection{Scenario 3-the engineer}

It is not typically financially possible to test every weld on every product, and therefore, it is necessary to shift the focus from the product towards the process. It would be desirable for the engineer to understand the factors influencing quality and make sure those factors are under control instead. A study performed by Hammersberg and Olsson [17] shows the possibility to identify important factors by using DOE (design of experiments). The study showed that weld toe radii, throat size and penetration requirements could all be fulfilled with certain parameter settings as illustrated in Fig. 8.

The surprising result was that the parameters mainly influencing the result were dimensional (angles and gap size) and not the commonly discussed parameters such as current

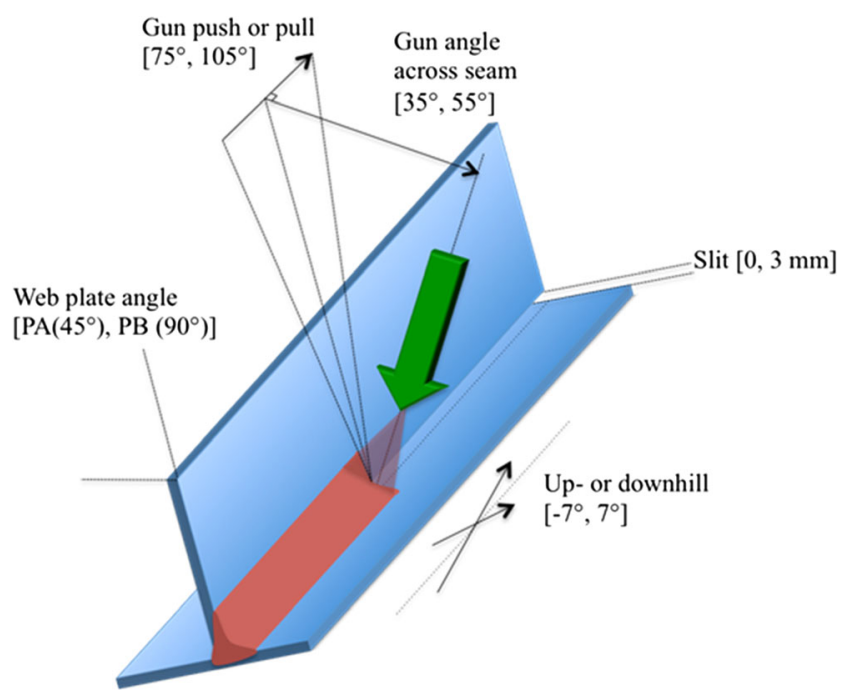

Fig. 8 The main factors for controlling the fillet weld dimensions according to Hammersberg and Olsson [17] and voltage. An uncoupled system, one set of parameters controlling the metallurgy and welding productivity and another set of parameters controlling the output geometry of the weld, indicates that indirect parameters could be measured instead. If as an example the angles could be measured instead of the radius, it means a totally different evaluation solution than what would be expected at first.

\subsection{Interview study}

An interview study was performed at 19 plants where welding is an important manufacturing process for creating the load-carrying structures. The persons interviewed held positions of welding coordinator, welding specialist, quality manager or similar. The questions were, in accordance with Lantz [18], semi-structured and the respondents had the chance to add more information during the interviews than was asked for.

The study shows that the current process for choosing evaluation method differs between the plants. The person needing the information to make decisions is often not the starting point in the process, but rather the engineers or the inspectors. The data collected and the information generated in the evaluation process are generally not optimized for decision-making. The focus was mainly on inspecting product quality rather than controlling process parameters. This often leads to a reactive behaviour where responsibilities fall between people. The cost of poor quality and the chosen quality strategy was generally not clear. In case such cost was followed up, only direct repair costs were included. To regularly evaluate the measurement process itself, by using structured measurement system analysis (MSA) procedures, was not a widely spread method among the respondents. 


\section{Discussion}

There is much to gain for businesses by using a pull approach in this matter. The cost of the inspection itself can be reduced - a more efficient evaluation. The example of the toe radius shows that adapting methods to the need can lead to less expensive methods. The initial difference in cost of equipment for a radius gauge, described in "Scenario 1 - the welder", in comparison with a scanning device in "Scenario 2-the programmer", is in the range of being 1000 times less expensive. More important is that a better match between information need and the information acquired could lead to more effective decision-making. To use an indirect approach, for example controlling angles instead of the radius, has a significant impact, as illustrated by "Scenario 3-the engineer". It will make the inspection cheaper and easier. The significant effect is, however, the possibility to control the process. By supplying the information needed to do that, a shift from product focus to process focus is enabled. The organizational awareness of variability and the strength to act within and between all functions increase when these concepts are commonly defined and visualized.

However, several organizational issues arise. It is a challenge to make this interesting to the experts. If you are employed as a specialist in a certain method, you are probably not happy narrowing down the need for that method.

This also creates a change of the qualification need. It is, for example, necessary for the organization to understand the influence of variation. The interview study showed that the main focus is on the product rather than the process creating the product. That means the dimension of variation gets lost. The control chart with the radii in Fig. 5 shows an example of the consequences of thinking average value rather than variation. On average, the weld toe radii passed the quality requirement $(0.6 \mathrm{~mm}$ with the limit $0.3 \mathrm{~mm}$ ) while actually almost $30 \%$ of the measured points were outside the limit. If the decisions are based on the average value, in this case, the consequences could be catastrophic.

When a potential method has been identified, it needs to be tested by making an MSA (measurement system analysis) to see if it is good enough or not for that purpose. The interviewed factories however use MSA only on rare occasions. The knowledge gap is essential. To use MSA can have several benefits. Of course, it is beneficial to know if the chosen evaluation method is good enough or not. An indirect result of performing MSA can, however, be an incentive for the organization to start reflecting about variation, boosting proactivity, making it possible to talk about capability and taking the spread in measurement results as a structural component into account when making decisions.

It is also surprising that the attention to design of experiments (DOE) is so low, considering its potential. In many cases, there are specialists in these Six Sigma methods in the company but they are too few to change the common awareness. It seems necessary to get a critical mass of people having the common language of MSA and DOE to make a change. The common language in a company is often money. Problems to express the cost of poor quality and choice of quality assurance in monetary terms can as well act as a barrier.

This change in culture requires cross-functional work, involving people from management, quality assurance, welding and engineering. The way that companies are commonly organized can be a barrier to this approach.

This means that the main challenges are not technical but rather connected with issues about organization, knowledge and common language. It can be easy for one individual to comprehend the concept but much more difficult to get the entire organization to understand. To take this aspect into account is crucial in development. If the main focus is solely on technical issues and not on information need, there is a risk that the main points get lost in the process.

As simple as it may sound, a change in starting point will influence both the choice of NDT method and visualization and thereby how efficient and effective the NDT solutions will be in the end.

\section{Summary and conclusions}

There is much to gain for businesses by using a pull approach when considering evaluation methods. Adapting the methods to the need often leads to more accurate, less expensive methods and improves organizational proactivity regarding variations. However, there are challenges in the way companies are organized as well as their level of knowledge about methods such as MSA, DOE and control charts. These methods need to be interesting to the experts.

Our conclusions are as follows:

- Differentiated NDT based on information need has a great potential.

- The challenges are mainly in non-technical terms such as organization, knowledge and common language.

This research has been performed in a welding context. Future research could show if the same ideas apply to other areas. The applicability to SMEs (small and medium-sized enterprises) would also be of interest since their organization is often different from that of large companies.

Acknowledgments The research work being the base of this paper has been partly funded by Vinnova and the Knowledge Foundation. A great contribution has been made by the staff at Volvo CE Arvika plant. 
Open Access This article is distributed under the terms of the Creative Commons Attribution 4.0 International License (http:// creativecommons.org/licenses/by/4.0/), which permits unrestricted use, distribution, and reproduction in any medium, provided you give appropriate credit to the original author(s) and the source, provide a link to the Creative Commons license, and indicate if changes were made.

\section{References}

1. Prasad J, Nair CGK (2008) Non-destructive test and evaluation of materials. Tata McGraw-Hill Publishing Company, New Delhi

2. Raj B, Jayakumar T, Thavasimuthu M (2002) Practical nondestructive testing, 2nd edn. Woodhead Publishing, Cambridge

3. Ericson Öberg A, Hammersberg P, Svensson L-E (2013) The right evaluation method - an enabler for process improvement. Paper presented at the International Conference on Joining Materials, Helsingør, Denmark, 5-8 May 2013

4. Ericson Öberg A (2013) A different view of quality assurance for fatigue loaded structures, in Barsoum Z (Ed.) Proceedings of the 2nd Swedish conference on design and fabrication of welded structures, Borlänge, Sweden, 9-10 October 2013

5. Jonsson B, Samuelsson J, Marquis GB (2011) Development of weld quality criteria based on fatigue performance. Weld World 55(11-12):79-88

6. Kassner M, Küppers M, Sonsino CM, Bieker G, Moser C (2010) Fatigue design of welded components of railway vehicles - influence of manufacturing conditions and weld quality. Weld in the World 54(9-10):R267-R278

7. Björk T, Samuelsson J, Marquis G (2008) The need for a weld quality system for fatigue loaded structures. Weld in the World 52(1-2):34-46
8. Lee C-H, Chang K-H, Jang G-C, Lee C-Y (2009) Effect of weld geometry on the fatigue life of non-load-carrying fillet welded cruciform joints. Eng Fail Anal 16(3):849-855. doi:10.1016/j. engfailanal.2008.07.004

9. Volvo Group (2011) STD 181-0004 Fusion welding - weld classes and requirements; life-optimized welded structures; steel, thickness $\geq 3 \mathrm{~mm}$. Volvo Group, Göteborg

10. Swedish Standard (2004) SS-EN ISO 5817:2004 welding: fusionwelded joints in steel, nickel, titanium and their alloys (beam welding excluded) - quality levels for imperfections

11. Pang HLJ (1993) Analysis of weld toe profiles and weld toe cracks. Int J Fatigue 15(1):31-36. doi:10.1016/0142-1123(93)90074-Z

12. Harati E, Ottosson M, Karlsson L, Svensson L-E (2014) Nondestructive measurement of weld toe radius using Weld Impression Analysis, Laser Scanning Profiling and Structured Light Projection methods. Proc First Int Conf Weld Non Destruct Test Tehran, Iran, 25-26

13. Danielsson M, Holgård J (2010) Improving analysis of key performance measures at four middle-sized manufacturing companies: moving focus from what has happened to what to do. diploma work no. 32/2010. Chalmers University of Technology, Gothenburg

14. Deming WE (1994) The new economics for industry, government, education, 2nd edn. MIT Press, Cambridge

15. Wheeler DJ (2009) Twenty things you need to know. SPS Press, Knoxville

16. Lindgren E, Stenberg T (2011) Quality inspection and fatigue assessment of welded structures. MSc thesis, KTH, Stockholm, Sweden

17. Hammersberg P, Olsson H (2013) Proactive control of weld dimensions in robotised MAG welding. In: Barsoum Z (ed) Proceedings of the 2nd Swedish conference on design and fabrication of welded structures. Borlänge, Sweden, pp 9-10

18. Lantz A (2007) Intervjumetodik, 2nd edn. Studentlitteratur, Lund [in Swedish] 